\title{
FINITE SETS OF AFFINE POINTS WITH UNIQUE ASSOCIATED MONOMIAL ORDER QUOTIENT BASES
}

\author{
ZHE LI, SHUGONG ZHANG, and TIAN DONG \\ School of Mathemathics, Key Lab. of Symbolic Computation \\ and Knowledge Engineering (Ministry of Education), \\ Jilin University, Changchun 130012, PR China *
}

\begin{abstract}
The quotient bases for zero-dimensional ideals are often of interest in the investigation of multivariate polynomial interpolation, algebraic coding theory, and computational molecular biology, etc. In this paper, we discuss the properties of zero-dimensional ideals with unique monomial order quotient bases, and verify that the vanishing ideals of Cartesian sets have unique monomial order quotient bases. Furthermore, we reveal the relation between Cartesian sets and the point sets with unique associated monomial order quotient bases.

Keywords: Monomial order quotient basis; Cartesian set; Zero-dimensional ideal.

2000 Mathematics Subject Classification: 13P10
\end{abstract}

\section{Introduction}

Let $\mathbb{F}$ be a field of characteristic zero, and suppose that $\mathbb{F}[\boldsymbol{x}]:=\mathbb{F}\left[x_{1}, \ldots, x_{d}\right]$ is the polynomial ring in $d$ variables over $\mathbb{F}$. A finite linearly independent set $\Theta$ of linear functionals, mapping $\mathbb{F}[\boldsymbol{x}]$ to $\mathbb{F}$, is said to admit an ideal interpolation scheme if

$$
\operatorname{ker} \Theta=\{p \in \mathbb{F}[\boldsymbol{x}]: \theta(p)=0 \text {, for all } \theta \in \Theta\}
$$

forms a zero-dimensional ideal, cf. 11, 11, 8, 24, 17]. It was shown in [9, 18] that this holds if and only if there exists a finite set $\Xi \subset \mathbb{F}^{d}$ and $D$-invariant finite-dimensional subspaces $Q_{\boldsymbol{\xi}} \subset \mathbb{F}[\boldsymbol{x}], \boldsymbol{\xi} \in \Xi$, such that

$$
\operatorname{span}_{\mathbb{F}} \Theta=\operatorname{span}_{\mathbb{F}}\left\{\delta_{\boldsymbol{\xi}} \circ q(D): q \in Q_{\boldsymbol{\xi}}, \boldsymbol{\xi} \in \Xi\right\},
$$

*lizhe200809@gmail.com,sgzh@jlu.edu.cn,dongtian@jlu.edu.cn 
where $D$ is the differentiation symbol and $\delta_{\boldsymbol{\xi}}$ denotes the point evaluation functional at $\boldsymbol{\xi}$.

We now assume that $\operatorname{ker} \Theta$ forms a zero-dimensional ideal. For an order ideal $O \subset \mathbb{F}[\boldsymbol{x}]$, if the set $\{\boldsymbol{t}+\operatorname{ker} \Theta: \boldsymbol{t} \in O\}$ provides an $\mathbb{F}$-vector space basis for quotient ring $\mathbb{F}[\boldsymbol{x}] / \operatorname{ker} \Theta$, then $O$ forms a monomial order quotient basis for ker $\Theta$. In many applications such as multivariate polynomial interpolation[21, algebraic coding theory [13, and computational molecular biology [16], the structure of monomial order quotient bases rather than Gröbner bases is of particular interest.

The standard example of ideal interpolation is Lagrange interpolation. In this case, $\operatorname{ker} \Theta=\mathcal{I}(\Xi)$, the vanishing ideal of $\Xi$, and a monomial order quotient basis $O$ for $\mathcal{I}(\Xi)$ is also called an associated monomial order quotient basis of $\Xi$. As is well known, the structure of $O$ depends not only on the cardinal but significantly on the geometry of $\Xi$, cf. [20, 23]. As interpolation point sets with special geometries, Cartesian sets in $\mathbb{F}^{d}$ have been studied by many authors 15, 12, 7, 6. In 2004, T. Sauer 22 showed that the vanishing ideal of a Cartesian set has a unique Gröbner éscalier, independent of the monomial order. The natural questions are: (1) Does a Cartesian set also have a unique associated monomial order quotient basis? (2) If so, is there any non-Cartesian set satisfying this property?

In this paper, we first introduce two criteria for determining whether a zerodimensional ideal has a unique monomial order quotient basis or not. With the aid of them, we show that every Cartesian set has a unique associated monomial order quotient basis, and furthermore that for $d \geq 3$, there always exists at least one non-Cartesian point set that also has this property. The main results of this paper will be put in Section 3. The next section, Section 2, will give some notation and background results.

\section{Notation and Background Results}

In this section, we will settle the key notation used throughout the paper and give some background results. For more details, we refer the reader to [15, 2, 3 , 4, 14.

We use $\mathbb{N}_{0}$ to stand for the monoid of nonnegative integers and boldface type for tuples with their entries denoted by the same letter with subscripts, for example, $\boldsymbol{\alpha}=\left(\alpha_{1}, \ldots, \alpha_{d}\right)$.

Henceforward, $\leq$ will denote the usual product order on $\mathbb{N}_{0}^{d}$, that is, for arbitrary $\boldsymbol{\alpha}, \boldsymbol{\beta} \in \mathbb{N}_{0}^{d}, \boldsymbol{\alpha} \leq \boldsymbol{\beta}$ if and only if $\alpha_{i} \leq \beta_{i}, i=1, \ldots, d$. A finite subset $\mathcal{A} \subset \mathbb{N}_{0}^{d}$ is lower if for every $\boldsymbol{\alpha} \in \mathcal{A}, \mathbf{0} \leq \boldsymbol{\beta} \leq \boldsymbol{\alpha}$ implies $\boldsymbol{\beta} \in \mathcal{A}$.

A monomial $\boldsymbol{x}^{\boldsymbol{\alpha}} \in \mathbb{F}[\boldsymbol{x}]$ is a power product of the form $x_{1}^{\alpha_{1}} \cdots x_{d}^{\alpha_{d}}$ with $\boldsymbol{\alpha} \in \mathbb{N}_{0}^{d}$. Denote by $\mathbb{T}(\boldsymbol{x}):=\mathbb{T}\left(x_{1}, \ldots, x_{d}\right)$ the monoid of all monomials in $\mathbb{F}[\boldsymbol{x}]$. A finite monomial set $O \subset \mathbb{T}(\boldsymbol{x})$ is called an order ideal if for every $\boldsymbol{t} \in O, \boldsymbol{t}^{\prime} \mid \boldsymbol{t}$ implies $\boldsymbol{t}^{\prime} \in O$. Further, the corner of an order ideal $O$ is

$$
\mathcal{C}[O]=\left\{\boldsymbol{t} \in \mathbb{T}(\boldsymbol{x}): \boldsymbol{t} \notin O, x_{i} \mid \boldsymbol{t} \Rightarrow \boldsymbol{t} / x_{i} \in O, 1 \leq i \leq d\right\} .
$$


For each fixed monomial order $\prec$ on $\mathbb{T}(\boldsymbol{x})$, a nonzero polynomial $f \in \mathbb{F}[\boldsymbol{x}]$ has a unique leading monomial $\mathrm{LM}_{\prec}(f)$, which is the greatest one appearing in $f$ w.r.t. $\prec$. For arbitrary $S \subset \mathbb{F}[\boldsymbol{x}]$, the set of leading monomials of all polynomials in $S$ is denoted by $\operatorname{LM}_{\prec}(S)$. According to [19, the monomial set

$$
\mathcal{N}_{\prec}(\mathcal{I}):=\mathbb{T}(\boldsymbol{x}) \backslash \mathrm{LM}_{\prec}(\mathcal{I})=\left\{\boldsymbol{x}^{\boldsymbol{\alpha}} \in \mathbb{T}(\boldsymbol{x}): \mathrm{LM}_{\prec}(f) \nmid \boldsymbol{x}^{\boldsymbol{\alpha}}, \forall f \in \mathcal{I}\right\}
$$

is the Gröbner éscalier of ideal $\mathcal{I}$ w.r.t. $\prec$. In fact, every Gröbner éscalier of $\mathcal{I}$ forms a monomial order quotient basis for $\mathcal{I}$.

Definition 1. 22, 6] A finite set $\Xi \subset \mathbb{F}^{d}$ of distinct points is Cartesian if and only if there exists a lower set $\mathcal{A} \subset \mathbb{N}_{0}^{d}$ and injective functions $y_{i}: \mathbb{N}_{0} \rightarrow \mathbb{F}, 1 \leq$ $i \leq d$, such that $\Xi$ can be written as

$$
\Xi=\left\{\left(y_{1}\left(\alpha_{1}\right), \ldots, y_{d}\left(\alpha_{d}\right)\right) \in \mathbb{F}^{d}: \boldsymbol{\alpha}=\left(\alpha_{1}, \ldots, \alpha_{d}\right) \in \mathcal{A}\right\} .
$$

$\Xi$ is also called an $\mathcal{A}$-Cartesian set.

For instance, point set $\Xi=\{(2.3,1.2),(4.7,1.2),(1.5,1.2),(2.3,0.2)\} \subset \mathbb{Q}^{2}$ is a $\{(0,0),(1,0),(2,0),(0,1)\}$-Cartesian set.

Theorem 1. 22] Let $\Xi \subset \mathbb{F}^{d}$ be an $\mathcal{A}$-Cartesian set, then Gröbner éscalier $\mathcal{N}_{\prec}(\mathcal{I}(\Xi))$ w.r.t. any monomial order $\prec$ is identical to

$$
\mathfrak{N}:=\left\{\boldsymbol{x}^{\alpha}: \boldsymbol{\alpha} \in \mathcal{A}\right\} .
$$

\section{Main Results}

\subsection{Zero-dimensional ideals with unique monomial order quotient bases}

In this subsection we will establish two criteria for zero-dimensional ideals with unique monomial order quotient bases. First, we need the following easy lemma.

Lemma 2. Suppose that the monomials in the finite set $T \subset \mathbb{T}(\boldsymbol{x})$ are linearly independent modulo an ideal $\mathcal{I} \subset \mathbb{F}[\boldsymbol{x}]$. For each $1 \leq i \leq m$, let $T_{i}$ be a subset of $T$ and let $V_{i}$ be the $\mathbb{F}$-vector space generated by $\left\{\boldsymbol{t}+\mathcal{I}: \boldsymbol{t} \in T_{i}\right\}$. Then $\bigcap_{i=1}^{m} V_{i}$ is generated by $\left\{\boldsymbol{t}+\mathcal{I}: \boldsymbol{t} \in \bigcap_{i=1}^{m} T_{i}\right\}$.

Proof. We will use induction on $m$, the number of the monomial sets, to prove the lemma. When $m=2$, for an arbitrary $\boldsymbol{u}+\mathcal{I} \in V_{1} \cap V_{2}$, there exist $k_{\boldsymbol{t}}, l_{\boldsymbol{t}}, k_{\boldsymbol{t}^{\prime}}, l_{\boldsymbol{t}^{\prime \prime}} \in \mathbb{F}$ such that

$$
\left(\sum_{\boldsymbol{t} \in T_{1} \cap T_{2}} k_{\boldsymbol{t}} \boldsymbol{t}+\sum_{\boldsymbol{t}^{\prime} \in T_{1} \backslash T_{2}} k_{\boldsymbol{t}^{\prime}} \boldsymbol{t}^{\prime}\right) \bmod \mathcal{I} \equiv \boldsymbol{u} \equiv\left(\sum_{\boldsymbol{t} \in T_{1} \cap T_{2}} l_{\boldsymbol{t}} \boldsymbol{t}+\sum_{\boldsymbol{t}^{\prime \prime} \in T_{2} \backslash T_{1}} l_{\boldsymbol{t}^{\prime \prime}} \boldsymbol{t}^{\prime \prime}\right) \bmod \mathcal{I}
$$

which implies that

$$
\left(\sum_{\boldsymbol{t} \in T_{1} \cap T_{2}}\left(k_{\boldsymbol{t}}-l_{\boldsymbol{t}}\right) \boldsymbol{t}+\sum_{\boldsymbol{t}^{\prime} \in T_{1} \backslash T_{2}} k_{\boldsymbol{t}^{\prime}} \boldsymbol{t}^{\prime}-\sum_{\boldsymbol{t}^{\prime \prime} \in T_{2} \backslash T_{1}} l_{\boldsymbol{t}^{\prime \prime}} \boldsymbol{t}^{\prime \prime}\right) \equiv 0 \bmod \mathcal{I} .
$$


Consequently, we have

$$
\boldsymbol{u} \equiv \sum_{\boldsymbol{t} \in T_{1} \cap T_{2}} k_{\boldsymbol{t}} \boldsymbol{t} \bmod \mathcal{I}
$$

Now, assume that the lemma is true for $m-1$, i.e., $\bigcap_{i=1}^{m-1} V_{i}$ is generated by $\left\{\boldsymbol{t}+\mathcal{I}: \boldsymbol{t} \in \bigcap_{i=1}^{m-1} T_{i}\right\}$. Since $V_{m}$ is generated by $\left\{\boldsymbol{t}+\mathcal{I}: \boldsymbol{t} \in T_{m}\right\}$ and $\bigcap_{i=1}^{m-1} T_{i} \subset T$, the statement for $m=2$ implies the lemma.

The following proposition presents the first criterion for zero-dimensional ideals with unique monomial order quotient bases whose proof uses the notion of elimination order. Actually, a monomial order on $\mathbb{T}(\boldsymbol{x})$ is called an elimination order for $x_{i}$ if the monomial $x_{i}$ is greater than all monomials in

$$
\mathbb{T}\left(\boldsymbol{x} \backslash x_{i}\right):=\mathbb{T}\left(x_{1}, \ldots, x_{i-1}, x_{i+1}, \ldots, x_{d}\right)
$$

w.r.t. this order. A typical example is the lexicographic order $\succ_{\text {lex }(i)}$ with

$$
x_{i} \succ_{\text {lex }(i)} \cdots \succ_{\text {lex }(i)} x_{d} \succ_{\text {lex }(i)} x_{1} \succ_{\text {lex }(i)} \cdots \succ_{\text {lex }(i)} x_{i-1} .
$$

Proposition 3. Let $\mathcal{I} \subset \mathbb{F}[\boldsymbol{x}]$ be a zero-dimensional ideal and let $O$ be a monomial order quotient basis for $\mathcal{I}$. Then this monomial order quotient basis is unique if and only if for each $\boldsymbol{x}^{\boldsymbol{\alpha}} \in \mathcal{C}[O]$, the set $\left\{\boldsymbol{x}^{\boldsymbol{\beta}}: 0 \leq \boldsymbol{\beta} \leq \boldsymbol{\alpha}\right\}$ is linearly dependent modulo $\mathcal{I}$.

Proof. $\Longrightarrow$ : For each $1 \leq i \leq d$, let $\prec_{i}$ be an elimination order for $x_{i}$. Then by the uniqueness of the monomial order quotient basis for $\mathcal{I}$, we have

$$
\mathcal{N}_{\prec_{1}}(\mathcal{I})=\cdots=\mathcal{N}_{\prec_{d}}(\mathcal{I})=O .
$$

Next, we will show that for each $\boldsymbol{x}^{\boldsymbol{\alpha}} \in \mathcal{C}[O],\left\{\boldsymbol{x}^{\boldsymbol{\beta}}: 0 \leq \boldsymbol{\beta} \leq \boldsymbol{\alpha}\right\}$ is linearly dependent modulo $\mathcal{I}$.

An arbitrary $\boldsymbol{x}^{\alpha}$ in $\mathcal{C}[O]$ gives rise to monomial sets

$$
O_{i}:=\left\{\boldsymbol{x}^{\boldsymbol{\beta}} \in O: \boldsymbol{x}^{\boldsymbol{\beta}} \prec_{i} \boldsymbol{x}^{\boldsymbol{\alpha}}\right\}, \quad 1 \leq i \leq d .
$$

For each $1 \leq i \leq d$, let $V_{i}$ be the $\mathbb{F}$-vector space generated by $\left\{\boldsymbol{t}+\mathcal{I}: \boldsymbol{t} \in O_{i}\right\}$. It follows from the definition of the elimination order for $x_{i}$ and (1) that

$$
\bigcap_{i=1}^{d} O_{i} \subseteq\left\{\boldsymbol{x}^{\boldsymbol{\beta}}: 0 \leq \boldsymbol{\beta}<\boldsymbol{\alpha}\right\} \subseteq O .
$$

For each $1 \leq i \leq d, \boldsymbol{x}^{\boldsymbol{\alpha}} \in \mathcal{C}[O]=\mathcal{C}\left[\mathcal{N}_{\prec_{i}}(\mathcal{I})\right]$ implies that the set $O_{i} \bigcup\left\{\boldsymbol{x}^{\boldsymbol{\alpha}}\right\}$ is linearly dependent modulo $\mathcal{I}$. That is to say, $\boldsymbol{x}^{\alpha}+\mathcal{I} \in V_{i}, 1 \leq i \leq d$. Therefore, $\boldsymbol{x}^{\boldsymbol{\alpha}}+\mathcal{I} \in \bigcap_{i=1}^{n} V_{i}$. Recalling Lemma 2, we know that $\bigcap_{i=1}^{n} V_{i}$ is generated by $\left\{\boldsymbol{t}+\mathcal{I}: \boldsymbol{t} \in \bigcap_{i=1}^{n} O_{i}\right\}$. Hence, $\left(\bigcap_{i=1}^{n} O_{i}\right) \bigcup\left\{\boldsymbol{x}^{\boldsymbol{\alpha}}\right\}$ is linearly dependent modulo $\mathcal{I}$. According to (5), $\left\{\boldsymbol{x}^{\boldsymbol{\beta}}: 0 \leq \boldsymbol{\beta} \leq \boldsymbol{\alpha}\right\}$ is linearly dependent module $\mathcal{I}$.

$\Longleftarrow$ : Suppose that there exists another monomial order quotient basis $O^{\prime} \neq$ $O$ for $\mathcal{I}$. Let $\boldsymbol{x}^{\alpha}$ be an arbitrary element in $O^{\prime} \backslash O$. We now have two cases to consider. 
Case I: $\boldsymbol{x}^{\boldsymbol{\alpha}} \in O^{\prime} \cap \mathcal{C}[O]$.

In this case, $\left\{\boldsymbol{x}^{\boldsymbol{\beta}}: 0 \leq \boldsymbol{\beta} \leq \boldsymbol{\alpha}\right\}$ is linearly dependent modulo $\mathcal{I}$. $\boldsymbol{x}^{\boldsymbol{\alpha}} \in O^{\prime}$ leads to $\left\{\boldsymbol{x}^{\boldsymbol{\beta}}: 0 \leq \boldsymbol{\beta} \leq \boldsymbol{\alpha}\right\} \subseteq O^{\prime}$ since $O^{\prime}$ is an order ideal, which implies that $O^{\prime}$ is linearly dependent modulo $\mathcal{I}$. This is a contradiction to our hypothesis that $O^{\prime}$ is a monomial order quotient basis for $\mathcal{I}$. Hence, $\boldsymbol{x}^{\boldsymbol{\alpha}} \notin \mathcal{C}[O]$.

Case II: $\boldsymbol{x}^{\alpha} \in O^{\prime} \backslash \mathcal{C}[O]$.

In this case, $x^{\alpha} \notin O \cup \mathcal{C}[O]$. It follows from the definition of $\mathcal{C}[O]$ that there exists $\boldsymbol{x}^{\gamma} \in \mathcal{C}[O]$ such that $\boldsymbol{x}^{\gamma} \mid \boldsymbol{x}^{\boldsymbol{\alpha}}$, which implies that $\boldsymbol{x}^{\gamma} \in O^{\prime}$, and hence $\left\{\boldsymbol{x}^{\boldsymbol{\beta}}: 0 \leq \boldsymbol{\beta} \leq \boldsymbol{\gamma}\right\} \subseteq O^{\prime}$. By our assumption, $\left\{\boldsymbol{x}^{\boldsymbol{\beta}}: 0 \leq \boldsymbol{\beta} \leq \boldsymbol{\gamma}\right\}$ is linearly dependent modulo $\mathcal{I}$, and consequently $O^{\prime}$ is linearly dependent modulo $\mathcal{I}$ which leads to a contradiction too. Therefore, we conclude that $\boldsymbol{x}^{\alpha} \notin O^{\prime} \backslash \mathcal{C}[O]$.

In sum, we can deduce that $\boldsymbol{x}^{\boldsymbol{\alpha}} \notin O^{\prime}$, which contradicts the hypothesis $\boldsymbol{x}^{\alpha} \in O^{\prime} \backslash O$.

As the main theorem of this paper, Theorem 4 characterizes a new criterion for zero-dimensional ideals with unique monomial order quotient bases by elimination orders.

Theorem 4. For each $1 \leq i \leq d$, let $\prec_{i}$ be an elimination order for $x_{i}$. Then for each zero-dimensional ideal $\mathcal{I}$, the following are equivalent:

(1) I has a unique monomial order quotient basis.

(2) For any two monomial orders $\prec$ and $\prec^{\prime}, \mathcal{N}_{\prec}(\mathcal{I})=\mathcal{N}_{\prec^{\prime}}(\mathcal{I})$.

(3) $\mathcal{N}_{\prec_{i}}(\mathcal{I}), 1 \leq i \leq d$, are identical.

Proof. $(1) \Rightarrow(2)$ : Since $\mathcal{I}$ has a unique monomial order quotient basis, $\mathcal{N}_{\prec}(\mathcal{I})$ and $\mathcal{N}_{\prec^{\prime}}(\mathcal{I})$ obviously coincide for any two monomial orders $\prec$ and $\prec^{\prime}$.

$(2) \Rightarrow(3)$ is trivial.

$(3) \Rightarrow(1)$ : Set

$$
O:=\mathcal{N}_{\prec_{1}}(\mathcal{I})=\cdots=\mathcal{N}_{\prec_{d}}(\mathcal{I}) .
$$

The arguments in the proof of Proposition 3 implies that for each $\boldsymbol{x}^{\boldsymbol{\alpha}} \in \mathcal{C}[O]$, $\left\{\boldsymbol{x}^{\boldsymbol{\beta}}: 0 \leq \boldsymbol{\beta} \leq \boldsymbol{\alpha}\right\}$ is linearly dependent module $\mathcal{I}$. Therefore, $O$ is the unique monomial order quotient basis for $\mathcal{I}$.

Lemma 5. Let $\mathcal{I} \subset \mathbb{F}[\boldsymbol{x}]$ be a zero-dimensional ideal, and let $G$ and $G^{\prime}$ be the reduced Gröbner bases for $\mathcal{I}$ w.r.t. monomial orders $\prec$ and $\prec^{\prime}$ respectively. Then $\mathcal{N}_{\prec}(\mathcal{I})=\mathcal{N}_{\prec^{\prime}}(\mathcal{I})$ if and only if $\operatorname{LM}_{\prec}(G)=\operatorname{LM}_{\prec^{\prime}}\left(G^{\prime}\right)$. Furthermore, if $\operatorname{LM}_{\prec}(G)=\operatorname{LM}_{\prec^{\prime}}\left(G^{\prime}\right)$, then $G=G^{\prime}$.

Proof. It is easy to see that $\mathcal{C}\left[\mathcal{N}_{\prec}(\mathcal{I})\right]=\operatorname{LM}_{\prec}(G)$. Then $\mathcal{N}_{\prec}(\mathcal{I})=\mathcal{N}_{\prec^{\prime}}(\mathcal{I})$ if and only if $\operatorname{LM}_{\prec}(G)=\operatorname{LM}_{\prec^{\prime}}\left(G^{\prime}\right)$. Moreover, assume that $\operatorname{LM}_{\prec}(G)=\operatorname{LM}_{\prec^{\prime}}\left(G^{\prime}\right)$. Then the first statement of this lemma implies $\mathcal{N}_{\prec}(\mathcal{I})=\mathcal{N}_{\prec^{\prime}}(\mathcal{I}):=O$. Therefore, for each pair $g \in G, g^{\prime} \in G^{\prime}$ satisfying $\operatorname{LM}_{\prec}(g)=\operatorname{LM}_{\prec^{\prime}}\left(g^{\prime}\right), g-g^{\prime} \in$ $\operatorname{span}_{\mathbb{F}} O \cap \mathcal{I}=\{0\}$ follows by the property of reduced Gröbner bases, which leads to the lemma. 
The following corollary follows immediately from Theorem 3.1 and Lemma 3.2 .

Corollary 6. Let $\mathcal{I} \subset \mathbb{F}[\boldsymbol{x}]$ be a zero-dimensional ideal and $G_{i}$ be the reduced Gröbner basis for $\mathcal{I}$ w.r.t. an elimination order $\prec_{i}$ for $x_{i}$. If $\operatorname{LM}_{\prec_{i}}\left(G_{i}\right), 1 \leq$ $i \leq d$, are identical, then $\mathcal{I}$ has a unique reduced Gröbner basis, independent of the monomial order.

Following T. Sauer 22, we refer to a reduced Gröbner basis $G$ for an ideal $\mathcal{I}$ as a universal Gröbner basis if $G$ is the unique reduced Gröbner basis for $\mathcal{I}$, independent of the monomial order.

Suppose that $\Theta$ gives an ideal interpolation scheme. We can compute $\mathcal{N}_{\prec_{i}}(\operatorname{ker} \Theta)$, $1 \leq i \leq d$, to decide whether $\operatorname{ker} \Theta$ has a unique monomial order quotient basis or not. If

$$
\Theta=\left\{\delta_{\boldsymbol{\xi}_{k}} \circ \frac{\partial^{\|\boldsymbol{\alpha}\|}}{\partial x_{1}^{\alpha_{1}} \ldots \partial x_{d}^{\alpha_{d}}}: \boldsymbol{\alpha} \in \mathcal{A}_{k}, 1 \leq k \leq \mu\right\},
$$

where $\boldsymbol{\xi}_{1}, \ldots, \boldsymbol{\xi}_{\mu} \in \mathbb{F}^{d}$ are distinct and $\mathcal{A}_{1}, \ldots, A_{\mu} \subset \mathbb{N}_{0}^{d}$ are lower, then we can use algorithms, such as the ones in [5, 10], to compare $\mathcal{N}_{\prec \text { lex }(i)}(\operatorname{ker} \Theta)$ without computing the Gröbner bases.

\section{Example 1. Let}

$$
\begin{array}{r}
\Theta=\left\{\delta_{(0,0,0)}, \delta_{(0,0,0)} \circ \frac{\partial}{\partial x_{3}}, \delta_{(0,0,0)} \circ \frac{\partial}{\partial x_{2}}, \delta_{(0,0,0)} \circ \frac{\partial}{\partial x_{1}},\right. \\
\left.\delta_{(0,0,0)} \circ \frac{\partial^{2}}{\partial x_{1} \partial x_{3}}, \delta_{(1,0,0)}, \delta_{(1,0,0)} \circ \frac{\partial}{\partial x_{3}}, \delta_{(0,0,1)}\right\} .
\end{array}
$$

A direct computation shows that

$$
\mathcal{N}_{\prec \text { lex }(i)}(\operatorname{ker} \Theta)=\left\{1, x_{1}, x_{1}^{2}, x_{2}, x_{3}, x_{3}^{2}, x_{1} x_{3}, x_{1}^{2} x_{3}\right\}, \quad i=1,2,3,
$$

which concludes that $\operatorname{ker} \Theta$ has a unique monomial order quotient basis.

\subsection{Vanishing ideals of Cartesian sets}

From Theorem 1 we see that the vanishing ideal of a Cartesian set has a unique Gröbner éscalier, independent of the monomial order. However, there also exists certain associated monomial order quotient basis of some point set which can not be Gröbner éscalier w.r.t. any monomial order.

For example, let $\Xi=\{(0,0),(1.1,-0.1),(0.1,0.9),(1,1)\}$ be a point set in $\mathbb{Q}^{2}$. Then $\left\{1, x_{1}, x_{2}, x_{1} x_{2}\right\}$ forms a monomial order quotient basis but not a Gröbner éscalier (w.r.t. any monomial order) of $\mathcal{I}(\Xi)$. Indeed, for an arbitrary monomial order $\prec$, we have either $x_{1}^{2} \prec x_{1} x_{2}$ or $x_{2}^{2} \prec x_{1} x_{2}$. Since both $\left\{1, x_{1}, x_{2}, x_{1}^{2}\right\}$ and $\left\{1, x_{1}, x_{2}, x_{2}^{2}\right\}$ are linearly independent modulo $\mathcal{I}(\Xi), x_{1}^{2}$ or $x_{2}^{2}$ must belong to $\mathcal{N}_{\prec}(\mathcal{I}(\Xi))$.

Next, we will show that Cartesian sets have unique associated monomial order quotient bases. To achieve this, we need to propose a criterion for multidimensional Cartesian sets, which is the extension of Theorem 5 of [ [ $]$ to general dimension. 
Specifically, for fixed $1 \leq i \leq d$, we cover a finite point set $\Xi \subset \mathbb{F}^{d}$ with exactly $m_{i}+1, m_{i} \in \mathbb{N}$, affine hyperplanes $l_{i, 0}, l_{i, 1}, \ldots, l_{i, m_{i}} \subset \mathbb{F}^{d}$ perpendicular to the $x_{i}$-axis. Moreover, set

$$
\begin{aligned}
\mathscr{L}_{i, j}(\Xi):= & \left\{\left(\xi_{1}, \ldots, \xi_{i-1}, \xi_{i+1}, \ldots, \xi_{d}\right):\right. \\
& \left.\forall\left(\xi_{1}, \ldots, \xi_{d}\right) \in \Xi \cap l_{i, j}\right\} \subset \mathbb{F}^{d-1}, \quad 1 \leq i \leq d, 0 \leq j \leq m_{i} .
\end{aligned}
$$

Without loss of generality, we assume that $\# \mathscr{L}_{i, 0}(\Xi) \geq \cdots \geq \# \mathscr{L}_{i, m_{i}}(\Xi)$.

Theorem 7. A finite set of distinct points $\Xi \subset \mathbb{F}^{d}, d \geq 2$, is Cartesian if and only if for each $1 \leq i \leq d$, we have

$$
\mathscr{L}_{i, 0}(\Xi) \supseteq \cdots \supseteq \mathscr{L}_{i, m_{i}}(\Xi)
$$

where $m_{i}$ and $\mathscr{L}_{i, j}(\Xi)$ are as above.

Proof. $\Longrightarrow$ : Recall (2). A Cartesian set $\Xi \subset \mathbb{F}^{d}$ can be written as

$$
\Xi=\left\{\left(y_{1}\left(\alpha_{1}\right), \ldots, y_{d}\left(\alpha_{d}\right)\right) \in \mathbb{F}^{d}: \boldsymbol{\alpha}=\left(\alpha_{1}, \ldots, \alpha_{d}\right) \in \mathcal{A}\right\}
$$

with lower set $\mathcal{A} \subset \mathbb{N}_{0}^{d}$ and injective functions $y_{i}: \mathbb{N}_{0} \rightarrow \mathbb{F}, 1 \leq i \leq d$.

Fix $1 \leq i \leq d$. For any $0 \leq \gamma_{i} \leq \beta_{i} \leq m_{i}$, according to (7), we have

$$
\begin{aligned}
\mathscr{L}_{i, \beta_{i}}(\Xi)=\{ & \left(y_{1}\left(\alpha_{1}\right), \ldots, y_{i-1}\left(\alpha_{i-1}\right), y_{i+1}\left(\alpha_{i+1}\right), \ldots, y_{d}\left(\alpha_{d}\right)\right): \\
& \left.\left(\alpha_{1}, \ldots, \alpha_{i-1}, \beta_{i}, \alpha_{i+1}, \ldots, \alpha_{d}\right) \in \mathcal{A}\right\}, \\
\mathscr{L}_{i, \gamma_{i}}(\Xi)=\{ & \left(y_{1}\left(\alpha_{1}\right), \ldots, y_{i-1}\left(\alpha_{i-1}\right), y_{i+1}\left(\alpha_{i+1}\right), \ldots, y_{d}\left(\alpha_{d}\right)\right): \\
& \left.\left(\alpha_{1}, \ldots, \alpha_{i-1}, \gamma_{i}, \alpha_{i+1}, \ldots, \alpha_{d}\right) \in \mathcal{A}\right\},
\end{aligned}
$$

which imply immediately that $\mathscr{L}_{i, \beta_{i}}(\Xi) \subseteq \mathscr{L}_{i, \gamma_{i}}(\Xi)$ for each $1 \leq i \leq d, 0 \leq \gamma_{i} \leq$ $\beta_{i} \leq m_{i}$.

$\Longleftarrow$ : Fix a point $\boldsymbol{\xi}=\left(\xi_{1}, \ldots, \xi_{d}\right)$ in $\Xi$. Let $l_{i, j}, 1 \leq i \leq d, 0 \leq j \leq m_{i}$, be covering hyperplanes defining $\mathscr{L}_{i, j}(\Xi)$. Thus, there must exist $\boldsymbol{\alpha} \in \mathbb{N}_{0}^{d}$ such that $\{\boldsymbol{\xi}\}=\bigcap_{i=1}^{d} l_{i, \alpha_{i}}$, where $0 \leq \alpha_{i} \leq m_{i}$ for all $1 \leq i \leq d$. Set

$$
\mathcal{A}:=\left\{\boldsymbol{\alpha} \in \mathbb{N}_{0}^{d}: \bigcap_{i=1}^{d} l_{i, \alpha_{i}} \in \Xi\right\} .
$$

It is easy to observe that $\Xi$ can be described as

$$
\Xi=\left\{\left(y_{1}\left(\alpha_{1}\right), \ldots, y_{d}\left(\alpha_{d}\right)\right):\left(\alpha_{1}, \ldots, \alpha_{d}\right) \in \mathcal{A}\right\},
$$

where $y_{i}: \mathbb{N}_{0} \rightarrow \mathbb{F}, 1 \leq i \leq d$, are certain injective functions. Let $\left(\alpha_{1}, \ldots, \alpha_{d}\right) \in$ $\mathcal{A}$. For any $\left(\beta_{1}, \ldots, \beta_{d}\right) \leq\left(\alpha_{1}, \ldots, \alpha_{d}\right)$ in $\mathbb{N}_{0}^{d}$, by (8), we have

$$
\left(y_{1}\left(\alpha_{1}\right), \ldots, y_{d-1}\left(\alpha_{d-1}\right)\right) \in \mathscr{L}_{d, \alpha_{d}}(\Xi) \subseteq \mathscr{L}_{d, \beta_{d}}(\Xi) .
$$


Thus, $\left(\alpha_{1}, \ldots, \alpha_{d-1}, \beta_{d}\right) \in \mathcal{A}$. Moreover,

$$
\left(y_{1}\left(\alpha_{1}\right), \ldots, y_{d-2}\left(\alpha_{d-2}\right), y_{d}\left(\beta_{d}\right)\right) \in \mathscr{L}_{d-1, \alpha_{d-1}}(\Xi) \subseteq \mathscr{L}_{d-1, \beta_{d-1}}(\Xi)
$$

implies $\left(\alpha_{1}, \ldots, \alpha_{d-2}, \beta_{d-1}, \beta_{d}\right) \in \mathcal{A}$, and so on. Finally, we can deduce that $\left(\beta_{1}, \ldots, \beta_{d}\right) \in \mathcal{A}$, which means that $\mathcal{A}$ is lower.

The next lemma and corollary establish a relation between a Cartesian set $\Xi \subset \mathbb{F}^{d}$ and its associated point sets $\mathscr{L}_{i, j}(\Xi) \subset \mathbb{F}^{d-1}$.

Lemma 8. Let $\Xi \subset \mathbb{F}^{d}, d \geq 2$, be an $\mathcal{A}$-Cartesian set, and let $m_{i}, \mathscr{L}_{i, j}(\Xi)$ be as in (8). For $1 \leq i \leq d, 0 \leq j \leq m_{i}$, set

$$
\begin{aligned}
\mathcal{A}_{i, j}:= & \left\{\left(\alpha_{1}, \ldots, \alpha_{i-1}, \alpha_{i+1}, \ldots, \alpha_{d}\right):\right. \\
& \left.\left(\alpha_{1}, \ldots, \alpha_{i-1}, j, \alpha_{i+1}, \ldots, \alpha_{d}\right) \in \mathcal{A}\right\} .
\end{aligned}
$$

Then $\mathscr{L}_{i, j}(\Xi)$ is $(d-1)$-dimensional $\mathcal{A}_{i, j}$-Cartesian for all $1 \leq i \leq d, 0 \leq j \leq m_{i}$.

Proof. Fix $1 \leq i \leq d, 0 \leq j \leq m_{i}$. For each $\left(\alpha_{1}, \ldots, \alpha_{i-1}, \alpha_{i+1}, \ldots, \alpha_{d}\right) \in$ $\mathcal{A}_{i, j}, \mathbf{0} \leq\left(\beta_{1}, \ldots, \beta_{i-1}, \beta_{i+1}, \ldots, \beta_{d}\right) \leq\left(\alpha_{1}, \ldots, \alpha_{i-1}, \alpha_{i+1}, \ldots, \alpha_{d}\right)$ implies that $\left(\beta_{1}, \ldots, \beta_{i-1}, j, \beta_{i+1}, \ldots, \beta_{d}\right) \in \mathcal{A}$. Then $\left(\beta_{1}, \ldots, \beta_{i-1}, \beta_{i+1}, \ldots, \beta_{d}\right) \in \mathcal{A}_{i, j}$ follows. Thus, $\mathcal{A}_{i, j} \subset \mathbb{N}_{0}^{d-1}$ is lower. By (9), (10) and (11), we can conclude that $\mathscr{L}_{i, j}(\Xi)$ is $\mathcal{A}_{i, j}$-Cartesian.

Corollary 9. Let $\Xi, \mathcal{A}$ and $\mathcal{A}_{i, j}$ be as in Lemma 8 , Define

$$
\begin{aligned}
\mathcal{A}_{i, j} \oplus j:= & \left\{\left(\alpha_{1}, \ldots, \alpha_{i-1}, j, \alpha_{i+1}, \ldots, \alpha_{d}\right):\right. \\
& \left.\forall\left(\alpha_{1}, \ldots, \alpha_{i-1}, \alpha_{i+1}, \ldots, \alpha_{d}\right) \in \mathcal{A}_{i, j}\right\} .
\end{aligned}
$$

Then for each $1 \leq i \leq d$, we have

$$
\mathcal{A}=\bigcup_{j=0}^{m_{i}} \mathcal{A}_{i, j} \oplus j .
$$

Proposition 10. Let $\Xi \subset \mathbb{F}^{d}$ be an $\mathcal{A}$-Cartesian set, and suppose that $\mathfrak{N}$ is as in (3). Then $\mathcal{I}(\Xi)$ has a unique monomial order quotient basis $\mathfrak{N}$.

Proof. We will prove this proposition by induction on $d$. When $d=1$, the proposition is obviously true. Suppose that for any $(k-1)$-dimensional Cartesian set, the statement holds.

Let $\Xi \subset \mathbb{F}^{k}$ be an $\mathcal{A}$-Cartesian set where $\mathcal{A} \subset \mathbb{N}_{0}^{k}$, and assume that $m_{i}$, $\mathscr{L}_{i, j}(\Xi)$ are as in (8) . For an arbitrary $\boldsymbol{x}^{\gamma} \in \mathcal{C}[\mathfrak{N}]$, there are two cases which must be examined.

Case I: $\gamma=\left(0, \ldots, 0, m_{k}+1\right)$.

Let $g\left(x_{k}\right)=\prod_{j=0}^{m_{k}} l_{k, j}$. Since $l_{k, 0}, l_{k, 1}, \ldots, l_{k, m_{k}}$ are affine hyperplanes covering $\Xi$, then $g\left(x_{k}\right) \in \mathcal{I}(\Xi)$ follows. Furthermore, it follows from the arguments in Theorem 7 that

$$
l_{k, j}=x_{k}-y_{k}(j), \quad j=0, \ldots, m_{k},
$$


which implies that $\left\{\boldsymbol{x}^{\boldsymbol{\beta}}: 0 \leq \boldsymbol{\beta} \leq \boldsymbol{\gamma}\right\}$ is linearly dependent modulo $\mathcal{I}(\Xi)$.

Case II: $\gamma=\left(\gamma_{1}, \ldots, \gamma_{k-1}, j\right)$ for some $0 \leq j \leq m_{k}$, and $x_{1}^{\gamma_{1}} \ldots x_{k-1}^{\gamma_{k-1}} \in$ $\mathcal{C}\left[\mathcal{N}_{k, j}\right]$ where

$$
\mathcal{N}_{k, j}=\left\{x_{1}^{\alpha_{1}} \cdots x_{k-1}^{\alpha_{k-1}}:\left(\alpha_{1}, \ldots, \alpha_{k-1}\right) \in \mathcal{A}_{k, j}\right\} .
$$

By inductive hypothesis, $\mathcal{N}_{k, j}$ is the unique monomial order quotient basis for $\mathcal{I}\left(\mathscr{L}_{k, j}(\Xi)\right)$, then there must exist $c_{\left(\alpha_{1}, \ldots, \alpha_{k-1}\right)} \in \mathbb{F}$ such that polynomial

$$
\hat{g}\left(x_{1}, \ldots, x_{k-1}\right)=x_{1}^{\gamma_{1}} \cdots x_{k-1}^{\gamma_{k-1}+} \sum_{\left(\alpha_{1}, \ldots, \alpha_{k-1}\right)<\left(\gamma_{1}, \ldots, \gamma_{k-1}\right)} c_{\left(\alpha_{1}, \ldots, \alpha_{k-1}\right)} x_{1}^{\alpha_{1}} \cdots x_{k-1}^{\alpha_{k-1}} \in \mathcal{I}\left(\mathscr{L}_{k, j}(\Xi)\right)
$$

by Proposition 3 . Set

$$
g(\boldsymbol{x}):=\hat{g}\left(x_{1}, \ldots, x_{k-1}\right) \prod_{i=0}^{j-1} l_{k, i},
$$

where the empty product is defined to be 1 . Since

$$
\mathscr{L}_{k, 0}(\Xi) \supseteq \cdots \supseteq \mathscr{L}_{k, m_{k}}(\Xi),
$$

it is easy to check that $\hat{g}\left(x_{1}, \ldots, x_{k-1}\right)$ vanishes at $\Xi \backslash\left\{\Xi \cap\left\{\cup_{i=0}^{j-1} l_{k, i}\right\}\right\}$ and $\prod_{i=0}^{j-1} l_{k, i}$ vanishes at $\Xi \cap\left\{\cup_{i=0}^{j-1} l_{k, i}\right\}$. Therefore, $g(\boldsymbol{x}) \in \mathcal{I}(\Xi)$. It follows that $\left\{\boldsymbol{x}^{\boldsymbol{\beta}}: 0 \leq \boldsymbol{\beta} \leq \boldsymbol{\gamma}\right\}$ is linearly dependent modulo $\mathcal{I}(\Xi)$.

In short, from Proposition 3 , we conclude that $\mathfrak{N}$ is the unique monomial order quotient basis for $\mathcal{I}(\Xi)$.

Finally, the following two theorems reveal the relation between Cartesian sets and the point sets with unique associated monomial order quotient bases.

Theorem 11. A finite set of distinct points $\Xi \subset \mathbb{F}^{2}$ is Cartesian if and only if it has a unique associated monomial order quotient basis.

Proof. Lemma 2.1 of [7] and Theorem 6 of [25] implies this theorem immediately.

Theorem 12. For every $d \geq 3$, there exists at least one non-Cartesian point set in $\mathbb{F}^{d}$ which has a unique associated monomial order quotient basis.

Proof. Let

$$
\Xi_{d}=\{(0,0,0, \underbrace{0, \ldots, 0}_{d-3}),(0,1,0, \underbrace{0, \ldots, 0}_{d-3}),(0,0,1, \underbrace{0, \ldots, 0}_{d-3}),(1,0,1, \underbrace{0, \ldots, 0}_{d-3})\}
$$

with $d \geq 3$. It is easy to check that $\left\{x_{1}\left(x_{1}-1\right), x_{2}\left(x_{2}-1\right), x_{3}\left(x_{3}-1\right), x_{1} x_{2}, x_{2} x_{3},\left(x_{3}-\right.\right.$ 1) $\left.x_{1}, x_{4}, \ldots, x_{d}\right\}$ forms a universal Gröbner basis for $\mathcal{I}\left(\Xi_{d}\right)$. Hence, Theorem 4 implies that $\Xi_{d}$ has a unique associated monomial order quotient basis. 


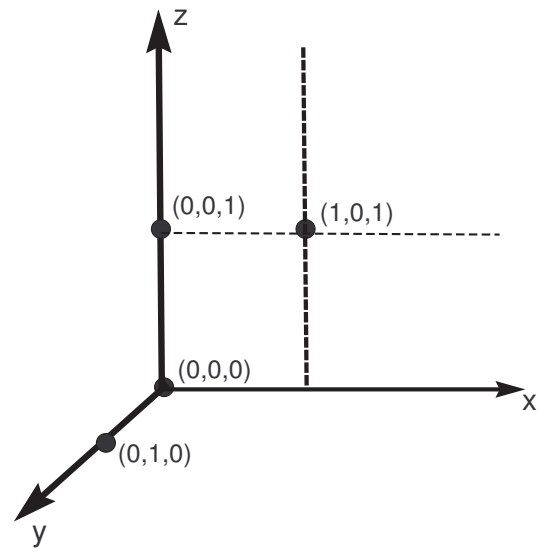

(a) $\Xi_{3}$



(b) $\left\{1, x_{3}, x_{2}, x_{1}\right\}$

Figure 1: $\Xi_{3}$ and its unique associated monomial order quotient basis

We now claim that $\Xi_{d}$ is non-Cartesian. To see this, we use induction on $d$. When $d=3, \Xi_{3}$ is illustrated in (a) of Figure 1 .

Recalling Theorem 7 , we can obtain

$$
\begin{aligned}
\mathscr{L}_{1,0}\left(\Xi_{3}\right) & =\{(0,0),(0,1),(1,0)\}, & & \mathscr{L}_{1,1}(\Xi)=\{(0,1)\}, \\
\mathscr{L}_{2,0}\left(\Xi_{3}\right) & =\{(0,0),(0,1),(1,1)\}, & & \mathscr{L}_{2,1}(\Xi)=\{(0,0)\}, \\
\mathscr{L}_{3,0}\left(\Xi_{3}\right) & =\{(0,0),(0,1)\}, & \mathscr{L}_{3,1}(\Xi) & =\{(0,0),(1,0)\} .
\end{aligned}
$$

We cover $\Xi_{3}$ with three sets of affine hyperplanes

$$
\left\{l_{1,0}, l_{1,1}\right\},\left\{l_{2,0}, l_{2,1}\right\},\left\{l_{3,0}, l_{3,1}\right\}
$$

where $l_{1,0}=x_{1}-0, l_{1,1}=x_{1}-1, l_{2,0}=x_{2}-0, l_{2,1}=x_{2}-1, l_{3,0}=x_{3}-0, l_{3,1}=$ $x_{3}-1$. Since $\mathscr{L}_{3,0}\left(\Xi_{3}\right) \nsupseteq \mathscr{L}_{3,1}\left(\Xi_{3}\right), \Xi_{3}$ is not Cartesian according to Theorem 7.

Assume that $\Xi_{d-1}$ is non-Cartesian. If $\Xi_{d}$ is Cartesian, then Lemma 8 also leads to a contradiction to our assumption.

\section{Acknowledgements}

The authors wish to thank the Reviewers for valuable suggestions and comments that have improved the presentation of the paper. 


\section{References}

[1] G. Birkhoff, The algebra of multivariate interpolation, in Constructive Approaches to Mathematical Models, eds. C. V. Coffman, G. J. Fix (Academic Press, 1979), pp. 345-363.

[2] B. Buchberger, Bruno Buchberger's PhD thesis 1965: An algorithm for finding the basis elements of the residue class ring of a zero dimensional polynomial ideal. J. Symb. Comput. 41(3-4) (2006) 475-511.

[3] B. Buchberger, Ein algorithmisches Kriterium fur die Lšbarkeit eines algebraischen Gleichungssystems (An Algorithmical Criterion for the Solvability of Algebraic Systems of Equations), Aequationes Math. 4(3) (1970) $374-383$.

[4] B. Buchberger, Gröbner-Bases: An Algorithmic Method in Polynomial Ideal Theory, Chapter 6 in: N.K. Bose (ed.), Multidimensional Systems Theory-Progress, Directions and Open Problems in Multidimensional Systems Theory (Reidel Publishing Company, Dodrecht-Boston-Lancaster, 1985) 184-232.

[5] L. Cerlienco and M. Mureddu, From algebraic sets to monomial linear bases by means of combinatorial algorithms, Discrete Math. 139(1995) 73-87

[6] T. Chen, T. Dong and S. Zhang, The Newton interpolation bases on lower sets, J. Inf. Comput. Sci. 3 (2006) 385-394.

[7] N. Crainic, Multivariate Birkhoff-Lagrange interpolation schemes and cartesian sets of nodes, Acta Math. Univ. Comenian.(N.S.) LXXIII (2004) $217-221$.

[8] C. de Boor, Ideal interpolation, in Approximation Theory XI: Gatlinburg 2004, eds. C. K. Chui, M. Neamtu, L. L. Schumaker(Nashboro Press, Brentwood TN, 2005) 59-91.

[9] C. de Boor and A. Ron, On polynomial ideals of finite codimension with applications to box spline theory, J. Math. Anal. Appl. 158(1991) 168-193.

[10] B. Felszeghy, B. Ráth and L. Rónyai, The lex game and some applications, J. Symb. Comput. 41 (2006) 663-681.

[11] M. Gasca and T. Sauer, Polynomial interpolation in several variables, Adv. Comput. Math., 12(2000), 377-410.

[12] M. Gasca and T. Sauer, On Bivariate Hermite Interpolation with Minimal Degree Polynomials, SIAM J. Numer. Anal., 37(3) (2000) 772-798.

[13] O. Geil, Algebraic geometry codes from order domains, Gröbner Bases, Coding, and Cryptography (Springer, Berlin, 2009) 121-142. 
[14] M. Kreuzer and L. Robbiano, Computational Commutative Algebra 2 (Springer, Berlin, 2005).

[15] R. Lorentz, Multivariate Birkhoff Interpolation, Lecture Notes in Math.1516 (Springer, Heidelberg, 1992).

[16] S. Lundqvist, Vector space bases associated to vanishing ideals of points, J. Pure Appl. Algebra 214 (2010) 309-321.

[17] Z. Li, S Zhang and T. Dong, On the existence of certain error formulas for a special class of ideal projectors, J. Approx. Theory doi:10.1016/j.jat.2011.02.011.

[18] M. G. Marinari, H. M. Möller and T. Mora, Gröbner bases of ideals defined by functionals with an application to ideals of projective points, Appl. Alg. Eng. Comm. Comput. 4(2) (1993) 103-145.

[19] T. Mora, Gröbner technology, Gröbner Bases, Coding, and Cryptography (Springer, Berlin, 2009) 11-25.

[20] T. Sauer and Y. Xu, On multivariate Lagrange interpolation, Math. Comp. 64 (1995) 1147-1170.

[21] T. Sauer, Polynomial interpolation of minimal degree, Numer. Math. 78(1) (1997) 59-85.

[22] T. Sauer, Lagrange interpolation on subgrids of tensor product grids, Math. Comp. 73 (2004) 181-190.

[23] T. Sauer. Polynomial interpolation in several variables: Lattices, differences, and ideals. in Multivariate Approximation and Interpolation, eds. M. Buhmann, W. Hausmann, K. Jetter, W. Schaback, and J. Stöckler(Elsevier B. V., 2006), pp. 189-228.

[24] B. Shekhtman, Ideal interpolation: Translations to and from Algebraic Geometry, in Approximate Commutative Algebra, eds. L. Robbiano, J. Abbott(Springer-Vienna, 2009), pp. 163-192.

[25] X. Wang, S. Zhang and T. Dong, A bivariate preprocessing paradigm for Buchberger-Möller algorithm, J. Comput. Appl. Math. 234 (2010) 33443355 . 